\title{
Sub-Picosecond Time-Resolved Spectroscopy of Energetic Materials: the Nitromethane and Nitro-Stilbenes
}

\author{
C. Rajchenbach, G. Jonusauskas and C. Rulliere \\ Centre de Physique Moléculaire Optique et Hertzienne (CPMOH), UA 283 du CNRS, Université de \\ Bordeaux I, 351 Cours de la Libération, 33405 Talence cedex, France
}

\begin{abstract}
Using sub-picosecond CARS experiment we observed direct photolysis of liquid nitromethane after pulse excitation at $299 \mathrm{~nm}$. We measured the dynamic behaviour under excitation of three main ground state Raman lines at $917 \mathrm{~cm}^{-1}, 1400 \mathrm{~cm}^{-1}$ and $2968 \mathrm{~cm}^{-1}$. We deduced an excited state lifetime of $1.1 \pm 0.3 \mathrm{ps}$ and we measured a photolysis quantum yield at $299 \mathrm{~nm}$ of $24 \% \pm 5 \%$. Using "pump-probe" experiments we observed the evolution of excited states of eight nitro-stilbene derivatives including 2,4,6,2',4',6'-hexanitro-stilbene (HNS). In the studied compounds important non-radiative desexcitation channels have been observed and their possible role on explosive process is discussed.
\end{abstract}

\section{INTRODUCTION:}

It has been already suggested [1] that molecular excited states may play a decisive role in ignition of detonation. It is then very important to observe the characteristics and the behaviour of molecular excited states. Comparison of excited state properties of explosive and non-explosive molecules may indeed allow to understand the specificity of explosive molecules and, finally, the key of the ignition of explosion and detonation processes. However, excited state lifetimes may be very short and these states, after excitation, may evolve and be transformed on a sub-picosecond time-scale. It is then necessary to observe these excited states with a sub-picosecond time-resolution.

To observe the behaviour of molecular excited states with sub-picosecond time-resolution two main techniques are available. The first one is "pump-probe" experiments in which one excites the sample with a UV pulse and observes its behaviour, after excitation, by means of a probe pulse. This method mainly involves electronic transitions between excited states. At certain wavelengths, corresponding to electronic transitions between excited states, the probe pulse can be absorbed by the different excited species present in the sample after excitation. The transmission characteristics (dynamic and spectral) of the probe pulse through the excited sample give information on the behaviour of the excited sample.

Rather than observe the behaviour of a sample under excitation through changes of electronic transitions, another way is to use a spectroscopic method to study the behaviour of certain vibrational modes of the sample. When a molecule is excited, we may expect to observe for example the disappearance or the evolution of its vibrational characteristics.

From this point of view, spontaneous and coherent Raman spectroscopies are powerful tools because it is well known that the Raman spectra of complex molecules give more detailed information and are more sensitive to structural changes in the molecules than other conventional spectroscopic methods such as absorption or fluorescence spectroscopy. In comparison with spontaneous Raman spectroscopy, coherent

+ Direction des Recherches et Etudes Techniques (DRET) fellowship 
anti-Stokes Raman scattering (CARS) offers two main advantages: The CARS signal is much stronger than the spontaneous Raman signal, and furthermore it is collimated in a well known and precise direction. Taking advantage of this, a CARS signal can be observed even in the presence of a strong luminescent or ambient light background and with high spectral resolution $[2,3]$. In this paper we describe applications of these two technics ("pump-probe" and CARS spectroscopy) to the study of excited states properties of explosive molecules: liquid nitromethane and nitro-stilbene derivatives.

Considerable experimental and theoretical work has been done on nitromethane in order to understand its photochemical and spectroscopic properties after UV excitation. Indeed, this rather small molecule, with well defined and intense Raman lines, is a unique model for testing and developing theoretical models, without involving large computations. Moreover nitromethane is the simplest of a series of explosive molecules. A better understanding of the explosion mechanism requires a detailed knowledge of the molecular properties under normal conditions before extrapolation to "explosive" conditions (high pressure and temperature) after shock initiation. It was then interesting to study this molecule under UV excitation. In this paper we describe, using sub-picosecond time-resolved CARS experiment, the observation of the decomposition of liquid nitromethane, after UV excitation, and measure the decomposition rate and the excited state lifetime.

HNS $\left(2,4,6,2^{\prime}, 4^{\prime}, 6^{\prime}\right.$-hexanitro-stilbene) is also a well known explosive and a member of the stilbene family. However trans-stilbene is not an explosive molecule. It is then interesting to progressively add nitro groups to this molecule and to transform trans-stilbene in mononitro, dinitro, tetranitro and finally hexanitro-stilbene (HNS). Comparison between the excited state properties of these different compounds could reveal some specificity related to the explosive characteristics of HNS for example. The possible existence of specificity could greatly improve the general understanding of ignition of detonation. In this paper we present some preliminary results concerning nitro-stilbene derivatives, including HNS, studied by "pump-probe" spectroscopy technics.

\section{EXPERIMENTAL SECTION:}

\subsection{CARS experimental set-up:}

The main part of this experimental set-up has been described elsewhere $[4,8]$. In the present work we used the degenerate (two-colour) CARS method which requires two beams: the pump beam at fixed frequency $\omega_{p}$ and the Stokes beam at variable frequency $\omega_{S}$ such that $\omega_{P}-\omega_{S}=\Omega$, the molecular vibration frequency to be observed. In such a situation the CARS signal is generated at frequency $\omega_{\text {CARS }}=\omega_{P}+\Omega=2 \omega_{P}-\omega_{S}$ in a different and well defined propagation direction with respect to the beams at frequencies $\omega_{p}$ and $\omega_{s}$. The propagation directions of the three beams $\left(\omega_{\mathrm{s}}, \omega_{\mathrm{p}}, \omega_{\mathrm{CARS}}\right)$ are in fact fixed by the phase matching condition imposed by the conservation of momentum. To excite the sample in the UV a third beam is necessary at frequency $\omega_{U V}$, which creates the excited population.

Our experiments were carried out using the following experimental set-up shown in figure 1. Our laser system employs a hybridly mode-locked dye laser (Coherent 702) which is synchronously pumped by the second harmonic of an actively mode-locked cw-pumped Nd3+:YAG laser (Coherent "Antares-76"). An active stabiliser (AS) (Coherent 7670 Active Amplitude and Position Stabiliser) improved the stability of the "Antares" output. The dye laser operates with rhodamine $6 \mathrm{G}$ solution as an amplifier medium and pinacyanol chloride in solution as a saturable absorber [5]. The dye laser output, centred at $598 \mathrm{~nm}$, $(0.5 \mathrm{~W} \mathrm{CW}$, pulse duration $\leq 500 \mathrm{fs}$ fwhm) is amplified by a dye amplifier ("Continuum PTA 60 " with Kiton Red 620 as amplifier dye) pumped by the frequency doubled output $(532 \mathrm{~nm}, 10 \mathrm{~Hz}, 70 \mathrm{ps}, 25 \mathrm{~mJ})$ of the Nd:YAG regenerative amplifier ("Continuum RGA 10"). The dye amplifier delivers pulses with an energy up to $1.5 \mathrm{~mJ}$ at a $10 \mathrm{~Hz}$ repetition rate. Dye amplifier output pulse parameters were controlled with a home-made single shot autocorrelator.

The dye amplifier output was frequency doubled in a KDP crystal to deliver the ultraviolet radiation at frequency $\omega_{\mathrm{UV}}(299 \mathrm{~nm}, \tau \leq 500 \mathrm{fs})$ used to excite the sample under study. The remaining part of the 598 nm beam, after the KDP crystal, was separated from the UV beam by means of a beam splitter. It is this beam which is used to create the beams at frequencies $\omega_{p}$ and $\omega_{S}$ and to generate the CARS signal. 


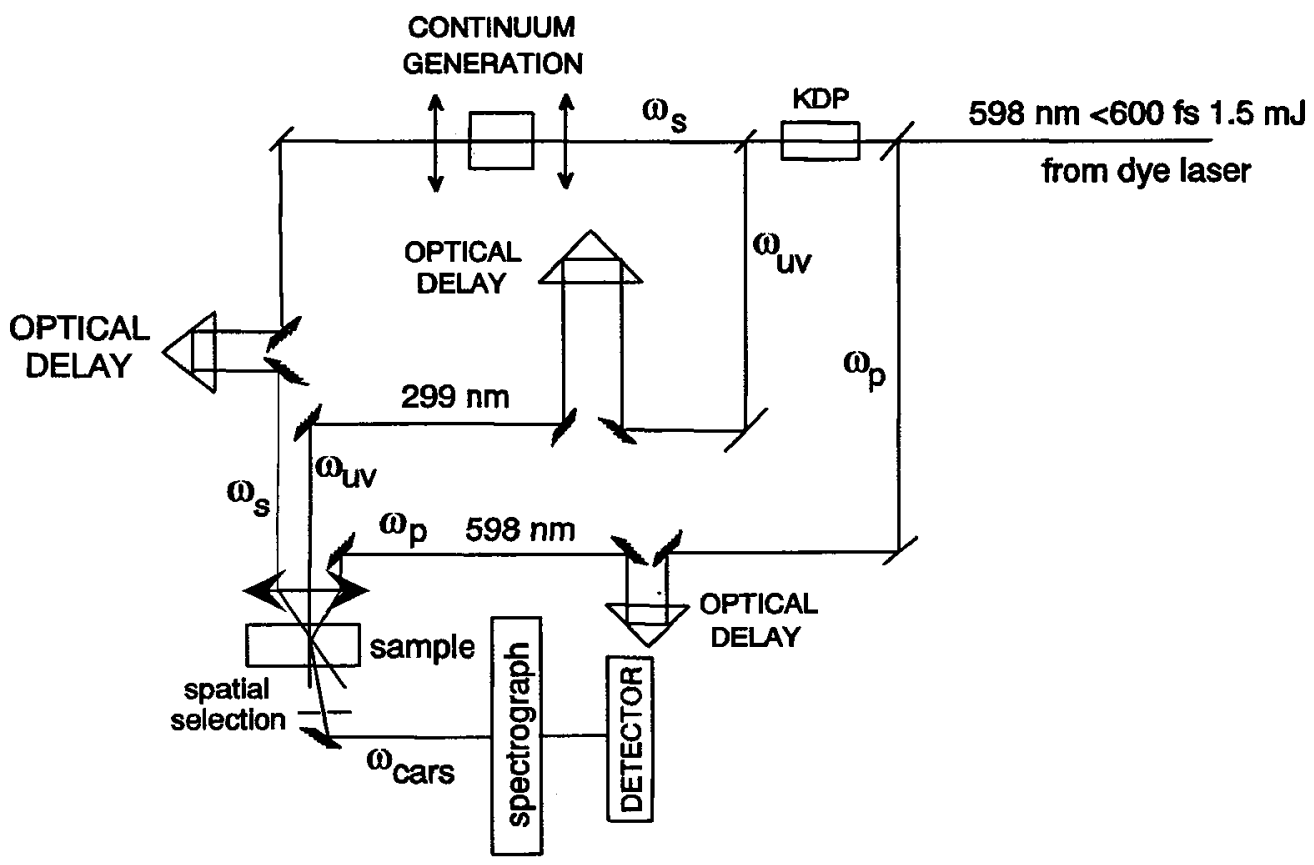

Fig.1: Experimental set-up of the time-resolved CARS experiments (see text).

Less than $10 \%$ of this $598 \mathrm{~nm}$ beam is used as the $\omega_{\mathrm{p}}$ beam in the CARS generation process. The rest is focused by a $10 \mathrm{~cm}$ focal lens into a rotating quartz disk or a $\mathrm{D}_{2} \mathrm{O}$ cell to produce a light continuum extending from $320 \mathrm{~nm}$ to $900 \mathrm{~nm}$. It is this continuum light which forms the Stokes beam at frequency $\omega_{\mathrm{S}}$, necessary for the CARS signal generation at frequency $\omega_{\mathrm{CARS}}=2 \omega_{\mathrm{p}}-\omega_{\mathrm{s}}$. Taking advantage of the large spectral width of this pulse, we were able to obtain the CARS spectrum over a wide spectral range (up to $2000 \mathrm{~cm}^{-1}$ ) and thus to observe simultaneously several Raman lines. The polarizations of the various incoming beams were adjusted in order to obtain maximum signal for the different vibration modes studied below.

The $\omega_{U V}, \omega_{p}$ and $\omega_{s}$ beams were focused into a nitromethane sample by a $15 \mathrm{~cm}$ focal length lens. The maximum energies were $5 \mu \mathrm{J} /$ pulse $\left(\omega_{\mathrm{p}}\right.$ beam), $10 \mu \mathrm{J} /$ pulse $\left(\omega_{\mathrm{s}}\right.$ beam in a $2500 \mathrm{~cm}^{-1}$ spectral range) and up to $50 \mu \mathrm{J}$ for the UV pump pulse ( $\omega_{\mathrm{UV}}$ pulse). The focused beam diameters at the sample were not more than $100 \mu \mathrm{m}$.

After spatial separation using an aperture, the CARS beam ${ }^{\omega}$ CARS was focused on the slit of a spectrograph (Chromex 5001S). Signal detection was achieved by an intensified photodiode-array (Princeton Instruments IRY 512 S/R) system connected to a computer for spectrum analysis.

Cross-correlation measurements of the pump and probe pulses are made using the optical Kerr effect in the nitromethane sample [6]. Using these procedures, the cross-correlation of the UV pump pulse and other pulses are measured to be shorter than $500 \mathrm{fs}$. Cross-correlation data also have been measured to ensure the synchronisation of all laser pulses to a zero delay at the sample with a maximum error of $60 \mathrm{fs}$. Delays between the UV pump and CARS generating pulses at the sample are achieved with an automatic translation stage (MT120 "Micro-Controle" with a 60 fs step unit). A second optical delay line allows control of the delay between the $\omega_{p}$ and $\omega_{s}$ beams and ensures perfect coincidence between these two pulses in the sample.

The experiments were then performed according to the following procedure. Without UV excitation of the sample, we optimised the CARS signal by adjusting positions and delays of the $\omega_{p}$ and $\omega_{s}$ beams and 
then recorded the CARS signal corresponding to the unexcited sample by accumulating 500 laser shots. Without any changes we sent the UV beam, and then recorded the CARS signal corresponding to the excited sample for a given time $\Delta t$ after excitation ( $\Delta t$ is determined by the respective positions of the different optical delay lines). Then we moved the optical delay line of the UV beam in order to change $\Delta t$ and again recorded the CARS signals corresponding to the unexcited and the excited sample. We made these series of experiments for different delay times $\Delta t$ and for different spectral ranges in order to study the behaviour of different Raman lines of the sample.

\subsection{Sub-picosecond "pump-probe" experiments:}

The main part of this experimental set-up has been described elsewhere $[7,8]$ and the laser system is the same as described above: hybridly modelocked dye laser (Coherent 702) synchronously pumped by the second harmonic of an actively mode-locked cw-pumped $\mathrm{Nd}^{3+}$ :YAG laser (Coherent "Antares-76") associated to regenerative amplifier (Continuum RGA 10) and dye amplifier (Continuum PTA 60).

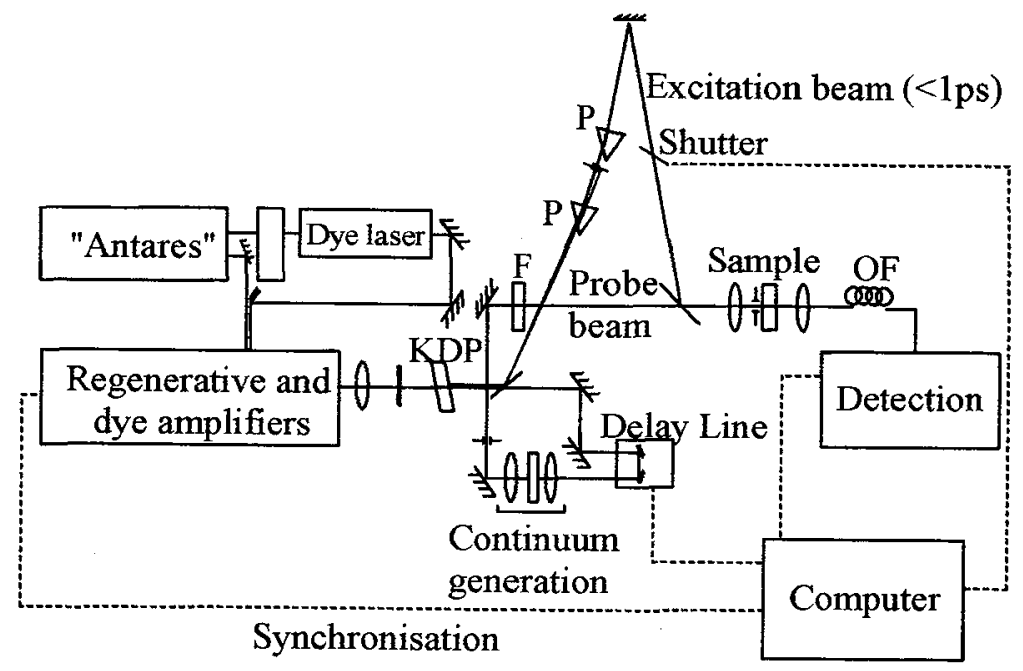

Fig.2: Experimental "pump-probe" set-up.

The pulses at $300 \mathrm{~nm}$ and $600 \mathrm{~nm}$ are separated by a beam splitter. The UV pulse $(300 \mathrm{~nm})$ is sent on the sample in order to excite the molecules to be studied. The $600 \mathrm{~nm}$ pulse, after passing through an optical delay line (60 fs steps) is focused on a rotating quartz plate to generate a continuum of light which extends from $320 \mathrm{~nm}$ to $900 \mathrm{~nm}$. This continuum is sent through the sample as the probe beam and analysed by means of a spectrograph (Chromex $5001 \mathrm{~S}$ ) and an intensified photodiode array (Princeton IRY 512 S/R) controlled by a computer. Measuring the spectral distribution of the continuum transmitted by the sample in presence and in absence of the UV pulse allows determination of transient absorption spectra of the sample. Changing the position of the optical delay line make possible observation of the time-evolution of the transient spectra (spectral shape and absorbance as a function of time) on a picosecond time-scale.

The UV pump pulse and the probe pulse were linearly polarised. By means of polarisers we adjusted to $54^{\circ} 7$ (the "magic angle") the angle between polarisation directions of these beams to ensure kinetics free from reorientational effects. 


\subsection{Samples:}

Commercially available nitromethane (Aldrich Chem., spectrophotometric grade) was used without further purification. A free flowing jet for the sample was used instead of a cell to avoid the accumulation of the photochemical products due to intense photo-irradiation and parasite signals from the windows. Moreover the small thickness of the jet $(\approx 200 \mu \mathrm{m})$ makes possible CARS signal generation in a large spectral range (up to $2000 \mathrm{~cm}^{-1}$ ) since in these conditions phase-matching conditions are not critical.

Nitro-stilbene derivatives used for these experiments are described below (see fig. 3) and the syntheses and purification will be described elsewhere [9-11]. Solvent was propylene carbonate (Aldrich Chemie, spectroscopic grade) and the concentrations of the order of $10^{-3} \mathrm{M} / \mathrm{l}$.

STILBENE

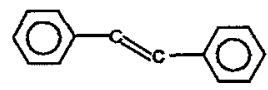

stilbene

NITROSTILBENE<smiles>O=[N+]([O-])c1ccccc1C=Cc1ccccc1</smiles>

2-nitrostilbene<smiles>C(C=Cc1ccccc1)=Cc1ccccc1</smiles>

3-nitrostilbene<smiles>O=[N+]([O-])c1ccc(C=Cc2ccccc2)cc1</smiles>

4-nitrostilbene
DINTTROSTLBENE<smiles>O=[N+]([O-])c1ccc(C=Cc2ccc([N+](=O)[O-])cc2)cc1</smiles>

4,4'-dinitrostilbene

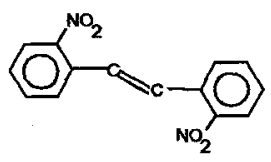

$2,2^{\prime}$-dinitrostilbene

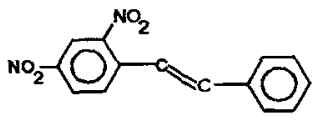

2,4-dinitrostilbene

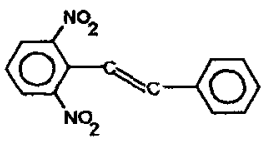

2,6-dinitrostilbene

\section{TETRANITROSTILBENE}<smiles>O=[N+]([O-])c1ccc(/C=C/c2ccc([N+](=O)[O-])cc2[N+](=O)[O-])c([N+](=O)[O-])c1</smiles>

$2,4,2^{\prime}, 4^{\prime}$-tetranitrostilbene<smiles>O=[N+]([O-])c1ccc(/C=C/c2ccc([N+](=O)[O-])c([N+](=O)[O-])c2)cc1</smiles>

$3,4,3^{\prime}, 4^{\prime}$-tetranitrostilbene

\section{HEXANITROSTILBENE}

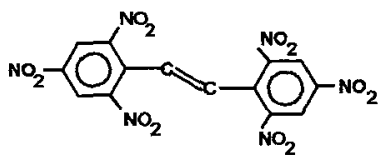

$2,4,6,2^{\prime}, 4^{\prime}, 6^{\prime}-$ hexanitrostilbene

Fig. 3: Studied stilbene and nitro-stilbene derivatives

\section{EXPERIMENTAL RESULTS AND DISCUSSION:}

\subsection{Nitromethane study by CARS technics:}

Some typical spectra recorded in the experimental conditions described above are shown in figure 4 . We observe that the CARS spectra are quite similar with and without UV excitation, with peaks centred near the following Raman lines of ground state nitromethane: the $v(\mathrm{CN})$ mode at $917 \mathrm{~cm}^{-1}$, the $v_{\mathrm{S}}\left(\mathrm{NO}_{2}\right)$ and $\delta_{\mathrm{S}}\left(\mathrm{CH}_{3}\right)$ modes near $1400 \mathrm{~cm}^{-1}$ and the $\nu_{\mathrm{S}}\left(\mathrm{CH}_{3}\right)$ mode at $2968 \mathrm{~cm}^{-1}$. Intensity changes are observed under UV excitation, but there are no new Raman lines which could be attributed to excited states of nitromethane. To summarise, with our experimental sensitivity only ground state Raman lines are observed. This point deserves some comment. It is indeed possible to observe Raman lines from excited states, as has been shown for various molecular species [11-16] and as we observed for example in t-stilbene [8]. However we should note that observation of excited state Raman lines was possible until now only when the Raman resonance conditions are fulfilled for the excited states to be studied but not for the ground state Raman lines. In other words the frequencies $\omega_{\mathrm{p}}$ and $\omega_{\mathrm{s}}$ should correspond to strong electronic transitions between excited states. In this case, Raman lines of the excited states are strongly enhanced with respect to those of the ground state, even if the excited state population is small compared to ground state population. In these conditions the CARS signal is a superposition of both the ground 
and the excited state Raman lines, the latter being strongly enhanced and quite obvious in the resultant CARS signal.
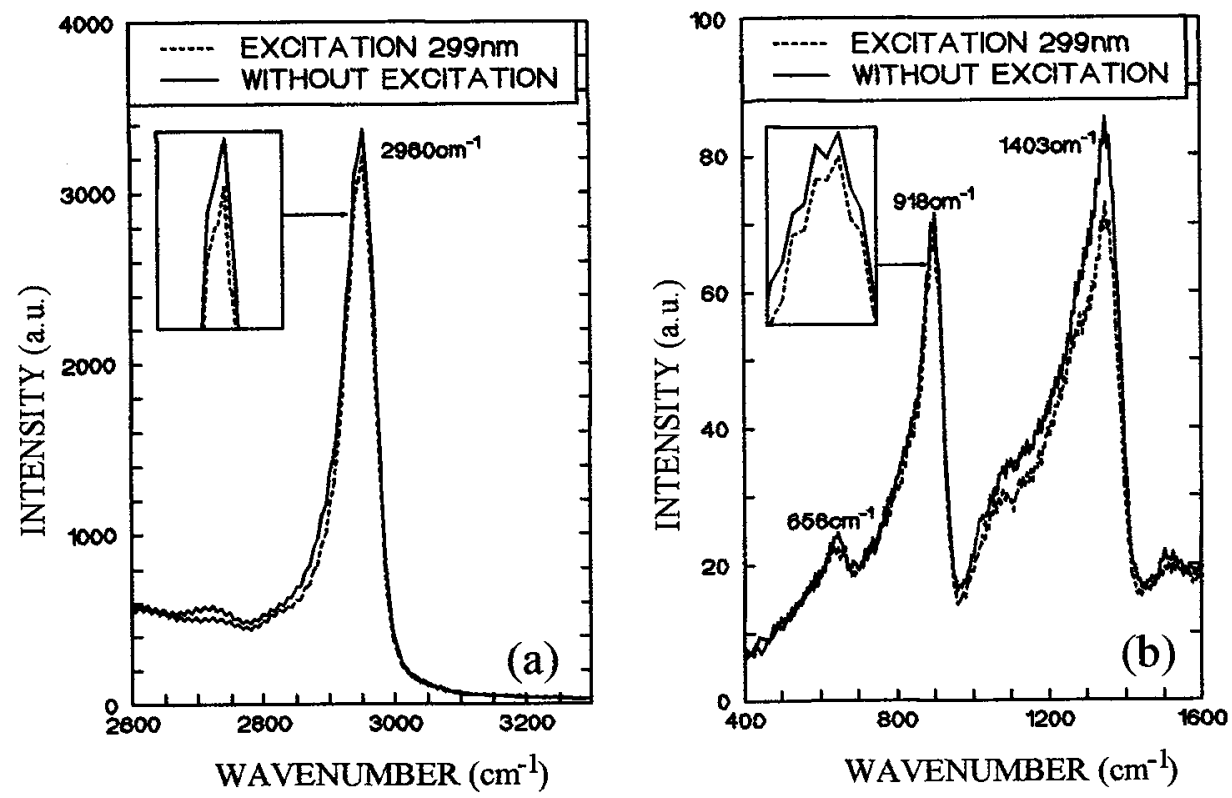

Fig. 4: Typical CARS spectra illustrating the observed changes under UV excitation. a) spectral range corresponding to the $v_{\mathrm{S}}\left(\mathrm{CH}_{3}\right)$ vibration mode near 2960 $\mathrm{cm}^{-1}$. The dotted spectrum (- - ) was obtained at time $\Delta \mathrm{t}=6 \mathrm{ps}$ after $\mathrm{UV}$ excitation. b) spectral range corresponding to the $\delta\left(\mathrm{NO}_{2}\right), v(\mathrm{CN}), \delta_{\mathrm{S}}\left(\mathrm{CH}_{3}\right)$ and $v_{\mathrm{S}}\left(\mathrm{NO}_{2}\right)$ vibration modes. The dotted spectra $(---)$ has been obtained at time $\Delta t=32$ ps after UV excitation.

To observe whether or not the resonance conditions was fulfilled in our experimental conditions, we made, as described in the introduction, "pump-probe" experiments using the experimental set-up described above. We did not detect any electronic transitions or absorbing transient species of excited nitromethane in the spectral range $\omega_{\mathrm{p}}$ and $\omega_{\mathrm{s}}$. That can be explained if we take into account that, due to the small size of nitromethane, electronic absorption bands are strongly shifted to the UV [17] in comparison with large size molecules (large size means at least ten carbon atoms). Such transitions should appear at larger frequencies than $\omega_{p}$ and $\omega_{s}$. We can thus deduce that our experimental conditions did not fulfil the resonance conditions for the excited states Raman lines. Due to these experimental conditions, Raman lines from the excited states did not appear on the CARS spectra. As a consequence the CARS spectra have to be considered as due to the Raman lines of the ground state molecules. Their evolution will thus give information about the evolution of the ground state population under UV excitation.

UV photo-excitation can perturb the ground state Raman lines in two ways. The first may involve some spectral changes (peak position, bandwidth) which can appear because of some changes affecting the ground state population under UV photo-excitation. Obviously figure 4 shows that no strong spectral changes are observed. Any spectral changes are smaller than the experimental error. The second way is a intensity change of the Raman lines which can be connected to variation of the ground state population. Under UV photo-excitation, the ground state can be depopulated (by transition from ground to excited states or photo-decomposition) or repopulated by electronic transitions (radiative or non-radiative) from excited to ground states. The intensity variations of the ground state Raman lines can thus be related to excited and ground state populations as described in detail in [18], after taking into account the non- 
resonant contribution to the CARS signal. It is possible, from the intensity of the Raman lines, to measure the kinetics of the ground state population. Some typical results are shown in figure $5 \mathrm{a}, \mathrm{b}$ and deserve some comments.
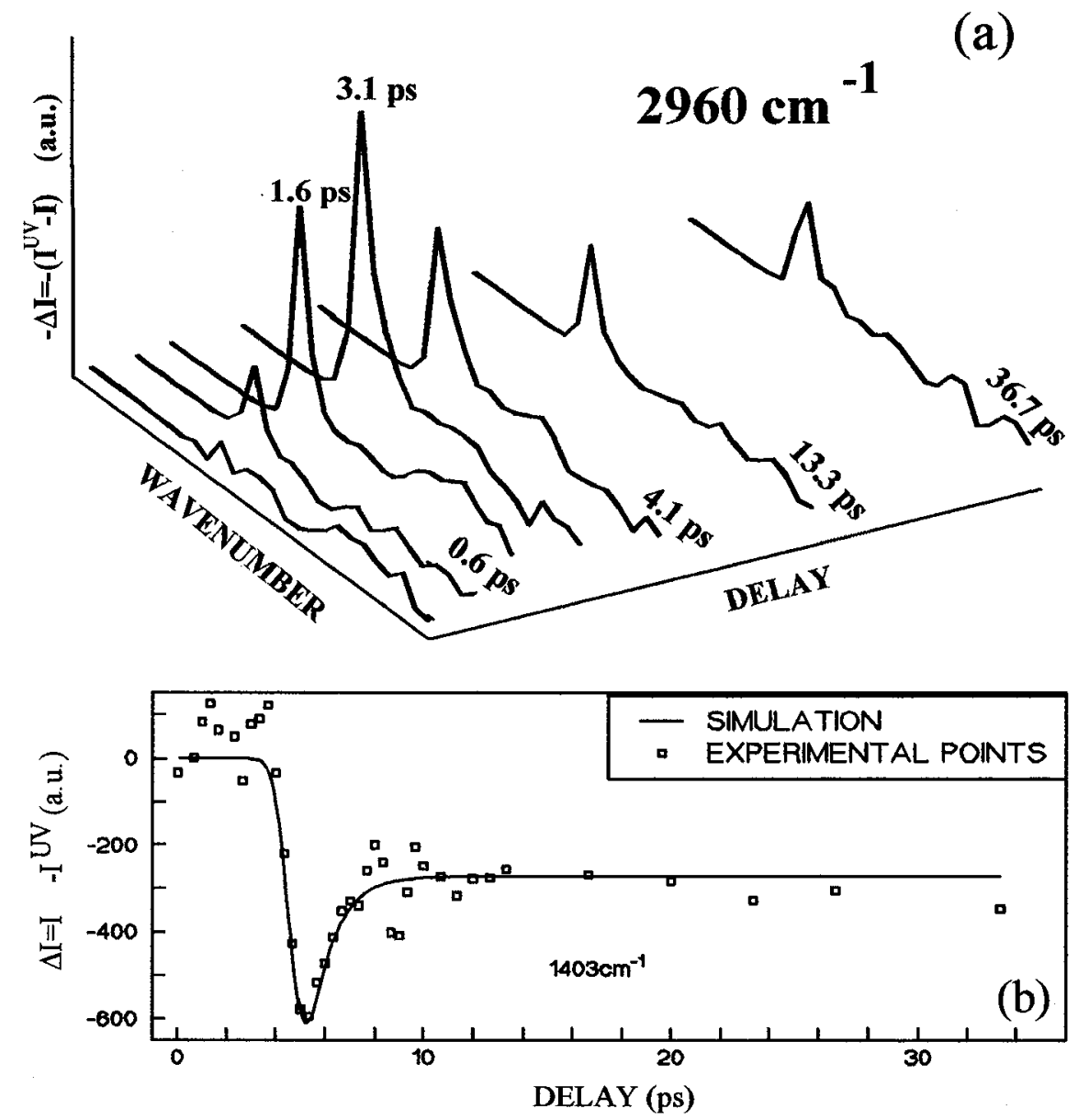

Fig. 5: a) $v_{\mathrm{S}}\left(\mathrm{CH}_{3}\right)$ vibrational mode intensity as a function of the time after $\mathrm{UV}$ excitation. Note that $\Delta \mathrm{I}$ values are negative and correspond to a decrease of the band intensity.

b) Difference of the integrated intensity of vibrational bands with and without UV excitation, $\Delta I$, as a function of delay after the UV pulse for the Raman lines near $1400 \mathrm{~cm}^{-1}$ $\left(\delta_{\mathrm{S}}\left(\mathrm{CH}_{3}\right)\right.$ and $\left.v_{\mathrm{S}}\left(\mathrm{NO}_{2}\right)\right)$.

The full line $(-)$ is the simulated curve. The results of the simulation are given in the text.

We observe that under excitation the CARS signal from the ground state of nitromethane sample immediately decreases, due to the depopulation of the ground state by the excitation pulse. A minimum intensity is reached corresponding to a maximum depopulation of the ground state. After this maximum has been reached, we observe a fast decay indicating that the ground state is repopulated from the excited state. Then the signal reaches a plateau which extends without change to $1 \mathrm{~ns}$, the longest time we could observe with our experimental set-up. The fast decay is an indication that the ground state population is 
quickly repopulated from the excited state. But the plateau indicates that after excitation the ground state population does not recover its initial value. In other words ground state nitromethane molecules have disappeared.

From these results we can infer that we observed the time-resolved decomposition of nitromethane. These results can be interpreted in the following way. During excitation a certain part of the ground state molecules is transformed in excited molecules. This creates a hole in the ground state population and a corresponding decreases of the CARS signal. After that, a part of the excited state population returns, by non-radiative transition to the ground state. This process is illustrated by the fast decay observed on the kinetics of figure 5. The other part of the excited state population will evolve on the photodecomposition energy surface pathway to new species. This part of the excited state population will never return to the ground state population, in agreement with the experimental lowering of the final CARS signal.

Taking into account these different processes we can model this behaviour to obtain the rate constants of the different processes by fitting the kinetic curves. The kinetic scheme can be illustrated as following:

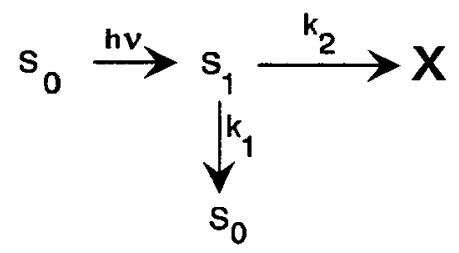

Scheme 1

where $S_{0}$ is the ground state, $S_{1}$ the excited state and $X$ the decomposition products. According to this scheme we were able to simulate these experimental curve [18] and to determine unambiguously the rate parameters $\mathrm{k}_{1}$ and $\mathrm{k}_{2}\left(1 / \mathrm{k}_{1}=1.5 \pm 0.3 \mathrm{ps}\right.$ and $\left.1 / \mathrm{k}_{2}=4.7 \pm 0.3 \mathrm{ps}\right)$. From these values we calculated an excited state lifetime of $1.1 \pm 0.3 \mathrm{ps}$ and a quantum yield of photo-decomposition $\left(\mathrm{k}_{2} /\left(\mathrm{k}_{1}+\mathrm{k}_{2}\right)\right)$ of $24 \pm 5 \%$ at $299 \mathrm{~nm}$. The results of the simulations are shown in figures $5 a$ and $b$ for the Raman lines at $1403 \mathrm{~cm}^{-1}$ and $2960 \mathrm{~cm}^{-1}$.

From these results some comments are in order with ignition of detonation. We observe that a fast decay channel from excited states to the ground state is observed. This channel is a non-radiative channel since the radiative lifetime, taking into account the absorption cross-section of the $S_{0} \rightarrow S_{1}$ transition and the nature of the excited state $\left(n-\pi^{*}\right)$ [19], is much longer than $10 \mathrm{~ns}$. This non-radiative channel may have some importance in the photo-decomposition of liquid nitromethane and more generally for decomposition under shock.

Indeed our results show that practically $76 \%$ of the energy deposited in the sample is evacuated non-radiatively. Excitation to the electronic state $S_{1}$ generates vibrationally hot ground state molecules by fast internal conversion of the photon excitation energy at $33445 \mathrm{~cm}^{-1}(\lambda=299 \mathrm{~nm})$. It has been shown that in this case temperature over $1000^{\circ} \mathrm{K}$ are reached by the molecules in their ground state $[20,21]$. In liquid nitromethane, taking into account the specific heat ( $C_{\mathrm{p}}=106.6 \mathrm{~J} / \mathrm{mol} \mathrm{K}$ [22]) and the excitation energy $\left(33444 \mathrm{~cm}^{-1}\right)$, a vibrational temperature of $3500 \mathrm{~K}$ can be theoretically reached. After that, the excess thermal energy is evacuated to the bath by collision with the neighbouring molecules of the first shell around the hot molecule. It has been shown $[20,21]$ that the molecules of the first shell also attain a very high temperature in some picoseconds, the rate of this thermalization process being controlled by the thermal conduction of the liquid. That means strong gradient temperature which will heat very fastly the different molecules after excitation. As a result a strong and fast local heating occurs by non-radiative transitions which can activate the decomposition. Since it has been shown that excited states are very important in the explosive process [1], we suggest that this process has to be taken into account for a microscopic description of the explosion mechanism on a microscopic scale. We will develop this point later and let us now present our results on nitro-stilbene derivatives.

\subsection{Study of nitro-stilbene derivatives:}

Figure 3 shows the studied compounds and figures 6 to 12 some typical transient absorption spectra recorded at different times after UV photoexcitation. The presented spectra are corrected for the influence of the chirp which appears between the spectral components of the probe continuum beam. In 
regard of the large number of studied compounds, it is difficult to explain in details the results obtained for each compound. That will be made in a forthcoming publication [23] and in this paper we will focus on the main important aspects of the photophysical behaviour of these compounds.

As explained in the introduction, the goal of this study is to try to observe a logical evolution of the photophysical properties of nitro-stilbenes, starting from $t$-stilbene and going to mono, di, tetra and finally hexanitro derivatives, in order to understand the specificity of nitro-stilbenes in relation with explosive ability. Let us then summarise the main tendencies deduced from our study.

First of all, in t-stilbene we did not observe any special behaviour from the observation of transient absorption spectra. A single absorption band was observed, centred near $580 \mathrm{~nm}$ and we did not note strong evolution of this band as a function of time. It was attributed to a single excited state, populated by excitation and decaying with a lifetime in the range of some tenths of picosecond. This is the classical behaviour of t-stilbene, already observed elsewhere [24].

If only one nitro group in introduced on the molecular skeleton of $t$-stilbene, the behaviour of $t$-stilbene becomes immediately different as shown in fig. 6 for 4-nitro-stilbene.

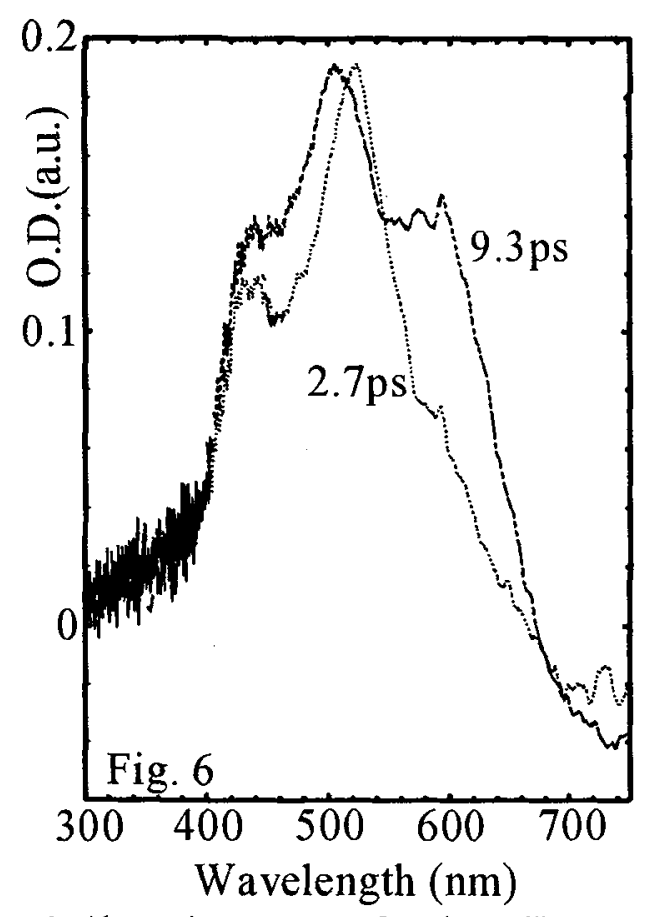

Fig. 6: Absorption spectra of 4-nitro-stilbene at different delay times after excitation. Dotted line: $2.7 \mathrm{ps}$; dashed line: $9.3 \mathrm{ps}$.

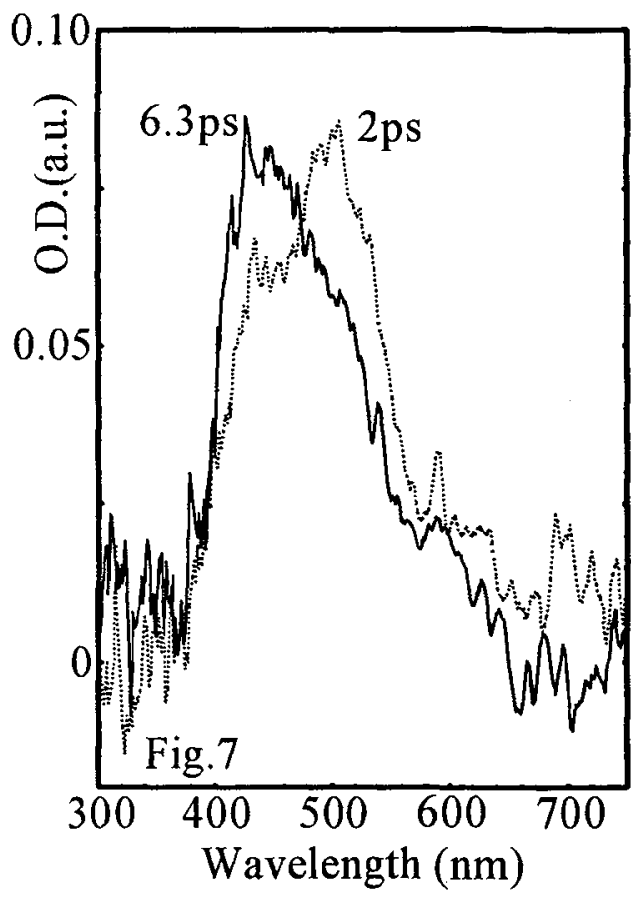

Fig. 7: Absorption spectra of 2-nitro-stilbene at different delay times after excitation. Full line: $6.3 \mathrm{ps}$; dotted line: $2.0 \mathrm{ps}$.

We may observe that three absorption bands can be observed developing on different time scales. These three different bands may be attributed to the presence at different times of at least three different excited species. Obviously the introduction of only one nitro group completely changes the photophysical behaviour of $t$-stilbene. It is interesting to observe how evolves this behaviour with the position of the nitro groups or with the introduction of more than one nitro group. Fig. 7 to 12 show some typical results. We may observe for example that the introduction at the ortho position of a nitro group changes the behaviour compared to introduction at the para position. In 2-nitro-stilbene (see fig. 7) only two absorption bands may be observed. One band (with maximum at $505 \mathrm{~nm}$ ) which appears with the excitation and a second band (with maximum at $440 \mathrm{~nm}$ ) which appears later. Obviously in this compound two excited states (related to absorption bands centred at $440 \mathrm{~nm}$ and at $505 \mathrm{~nm}$ ) are present, the first one being directly populated from the UV excitation pulse and the second one being populated 
from the first one in approximately $5 \mathrm{ps.} \mathrm{At} \mathrm{longer} \mathrm{times} \mathrm{(}>5 \mathrm{ps})$ the populations of these two states are in equilibrium. The comparison between 4-nitro-stilbene and 2-nitro-stilbene then shows that the position of the substitution may also change strongly the photophysical behaviour of these derivatives.

The influence of the introduction of more than one nitro group also changes drastically the photophysicai properties of nitro-stilbenes as illustrated on the next figures.

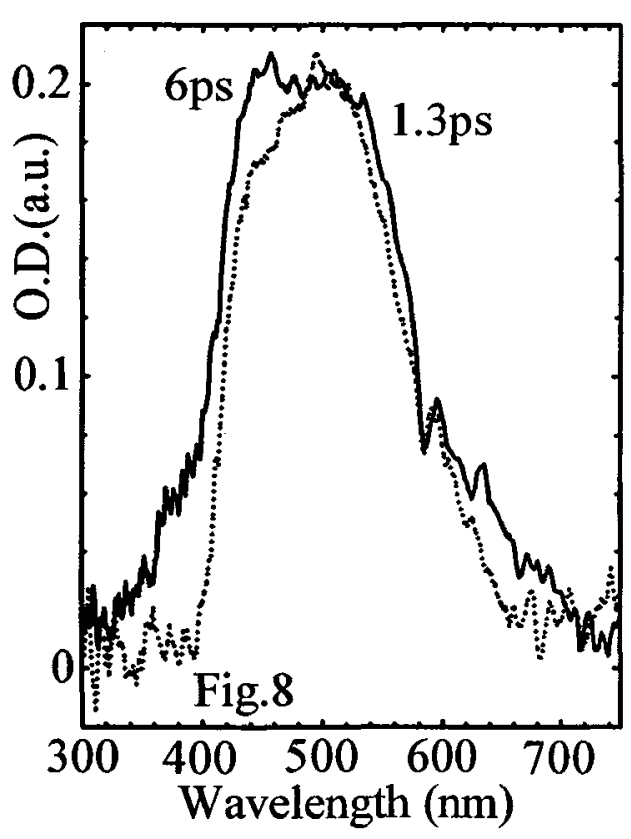

Fig.8: Absorption spectra of 2,4-dinitrostilbene at different delay times after excitation. Full line: $6.0 \mathrm{ps}$; dotted line: $1.3 \mathrm{ps}$.

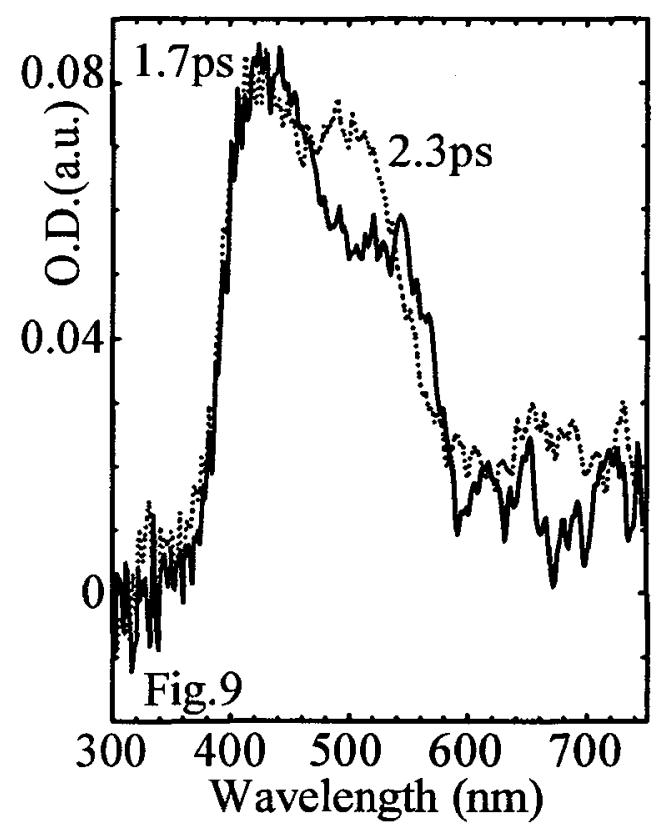

Fig.9: Absorption spectra of 2,2'-dinitrostilbene at different delay times after excitation. Full line: $1.7 \mathrm{ps}$; dotted line: $2.3 \mathrm{ps}$.

For example, in di-nitro derivatives we observed in all the studied compounds, the presence of two excited states (revealed by the presence of two absorption bands as shown in fig. 8 and 9). The populations of these two states are in equilibrium.

In tetra-nitro derivatives, as shown in fig. 10 and 11 , at least two states are still present but the equilibrium between their populations is strongly displaced in favour of last formed state. In 3,4,3',4'tetra-nitrostilbene, for example, at longer times than $5 \mathrm{ps}$, only the second excited state is populated and decays on a nanosecond time scale. The first created excited state is no longer populated. 


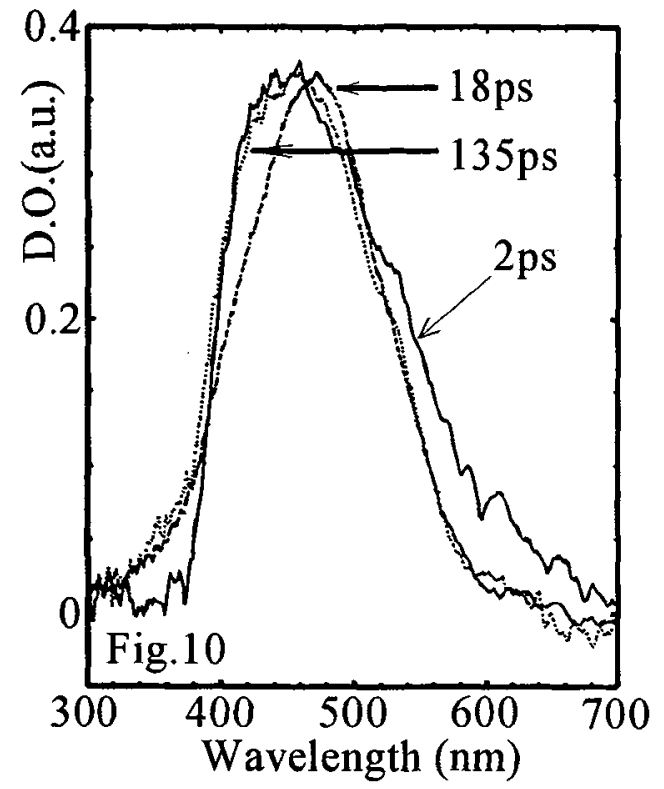

Fig.10: Absorption spectra of 2,4,2', $4^{\prime}$ tetranitrostilbene at different delay times after excitation. Full line: $2.0 \mathrm{ps}$; dotted line: $135 \mathrm{ps}$; dash line: 18ps.

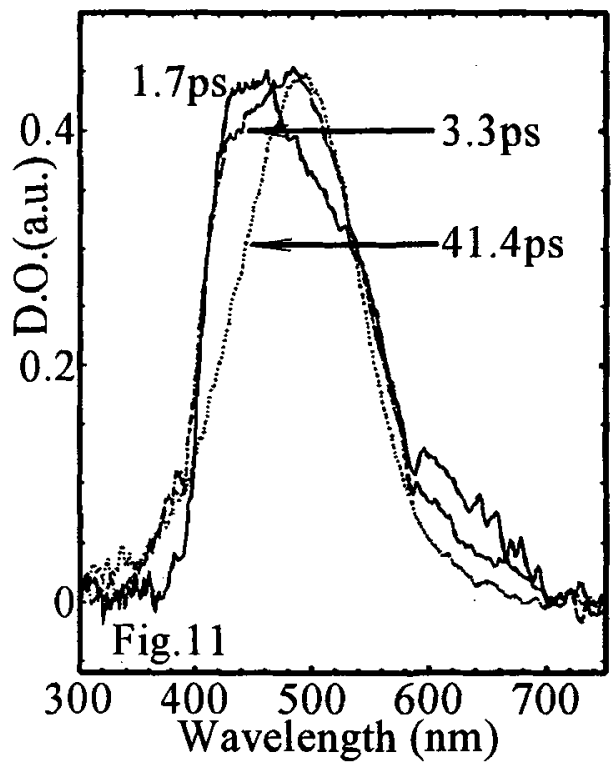

Fig. 11: Absorption spectra of $3,4,3^{\prime}, 4^{\prime}$ tetranitrostilbene at different delay times after excitation. Full line: $1.7 \mathrm{ps}$; dotted line: $41.4 \mathrm{ps}$; dash line: $3.3 \mathrm{ps}$

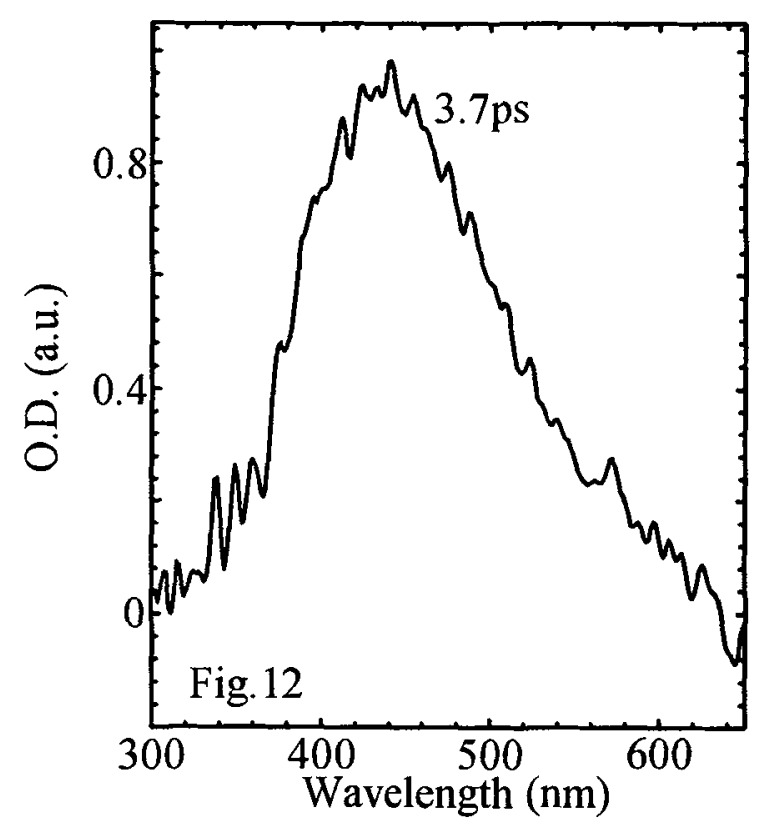

Fig.12: Absorption spectra of 2,4,6,2',4',6'-hexanitro-stilbene at different delay times after excitation. Full line: $3.7 \mathrm{ps}$. 
In $2,4,6,2^{\prime}, 4^{\prime}, 6^{\prime}$-hexanitro-stilbene, the first band is practically absent and not observed even at very short times after excitation. The second band appears fastly, is very broad and structureless and decays with a lifetime in the nanosecond time scale. No equilibrium is present in this compound.

Let us now summarise these different results. Introduction of nitro groups completely modifies the photophysical behaviour of t-stilbene with the appearance of several excited species which are not present in t-stilbene. In 4-nitro-stilbene, three absorption bands are observed, the second one shifting to the blue as a function of time in polar solvents. This shift is an indication of a relaxation process which may involve a molecular conformation change or a cage relaxation process. In any case this state is further stabilised by interaction with environment. It can be a possible indication that charge transfer exists in this compound. In fact comparison of the behaviour of 4-nitro-stilbene in polar and non-polar solvents [24] supports strongly this hypothesis.

If the number of nitro groups is greater than one there exist two excited species (excepted in $2,4,2^{\prime}, 4^{\prime}-$ tetra-nitro-stilbene) revealed by the observation of two different absorption bands evolving with different characteristic times. In any compounds the first state is immediately populated by the excitation pulse. This state is the franck-condon state accessible from the ground state configuration. From this first populated excited state, a second excited state is fastly populated with characteristic rise times in the range of 1-10 ps. Depending on the compound an equilibrium, more or less important, may exist between the populations of these states. This second state has a lifetime in the range of 1-5 nanosecond. When the number of nitro groups is equal or larger than four, the equilibrium practically does not exist. In HNS, this second excited state is very fastly populated after excitation.

Let us now discuss the specificity of HNS in regard of explosive ability taking into account these results. It seems reasonable to assert the explosive ability to the presence of the second excited state. This excited state seems to be the key of the explosive ability. Indeed, as the number of nitro groups is increasing this state is more and more populated after excitation. The equilibrium existing between the populations of the two observed excited states is strongly moved in favour of the second excited state. Moreover as the number of nitro groups increases this state is fastly populated, particularly in HNS, where the presence of the first excited state is difficult to detect with our available time-resolution.

If we accept that strong charge transfer exists in this state, it is reasonable to consider that charges are strongly localised on electron acceptor nitro groups. So the explosive ability can be correlated to this charge localisation. It's exactly the same conclusion that obtain Delpuech [1] on a theoretical basis. However before to conclude about the validity of this hypothesis other experiments are necessary. Let us however discuss and propose a possible mechanism for ignition of explosion under shock, in the light of our results.

It is well known that under shock, excited states can be populated in certain materials. This process is well known and called "mechano luminescence" [25-28]. To our knowledge this process has not been observed in nitromethane and nitro-stilbenes or more generally in explosives. But we have to take into account that, due to fast non-radiative channels, fluorescence quantum yield are very small and luminescence will be difficult to detect. It is then well possible that after shock excited states are formed. After excitation molecules may be decomposed as in nitromethane, the decomposition rate being increased by the local heating due to fast non-radiative transitions which create large gradient temperature. After these preliminary decompositions chain chemical reaction will be initiated opening the door to the detonation and explosion. In the case of nitro-stilbenes, it seems that it is the second excited state formed after excitation that is responsible for this process. In the case of these molecules we have to take into account the presence of fast non-radiative channels. For example the formation of the second excited state from the first one is very fast (in the range 1-10ps) and is also non-radiative and heat dissipative. Like in nitromethane we may imagine that fast local heating occurs and accelerates the decomposition reaction.

Naturally this proposed model is hardly speculative but it is quite possible to verify the validity of the developed points above.

1/ The spectra of excited states being now known, it is possible to envisage to check the presence of such states under shock and detonation conditions, for example by time-resolved absorption methods. These spectra are indeed the signature of these particular excited states: the observation or the non-observation of these spectra under shock could allow to test the validity of the proposed model.

2/ Fast non-radiative transitions seem still present in all the studied molecules in the present work. Nevertheless it will be also interesting to observe, like in nitromethane, if fast non-radiative transitions 
spectra are indeed the signature of these particular excited states: the observation or the non-observation of these spectra under shock could allow to test the validity of the proposed model.

2/ Fast non-radiative transitions seem still present in all the studied molecules in the present work. Nevertheless it will be also interesting to observe, like in nitromethane, if fast non-radiative transitions exist too in nitro-stilbenes as direct desexcitation channel between excited and ground state. Indeed we observed only fast non-radiative channel between excited states in nitro-stilbenes. We planify experiments to observe direct and fast non-radiative channel between excited and ground states. If such a channel really exists in these compounds, this fact could be one of the keys explaining explosive ability of such compounds.

\section{CONCLUSION:}

Use of time-resolved CARS and "pump-probe" experiments provides the first time-resolved data on the excited state behaviour of liquid nitromethane and nitro-stilbene derivatives. Very fast photochemical and photophysical channels have been revealed in the range of $1-10 \mathrm{ps}$, depending on the compounds. These experiments have also shown for the first time the presence, in nitromethane molecule, of a very important and fast non-radiative channel between the excited and the ground states. The presence of this channel means that most times that a molecule is excited, a strong and fast local heating occurs for the neighbouring molecules. The presence of this non-radiative channel has to be taken into account to explain the first steps of explosion on a microscopic level.

From these different data we proposed a hardly speculative model to explain the mechanism of detonation and explosion ignition on a microscopic level. We proposed some experiments to test the validity of this speculative model. Some of the proposed experiments are currently in progress in the lab.

Acknowledgements: This work was financially supported by Direction des Recherches et Etudes Techniques (DRET contract $\mathrm{N}^{\circ}$ 940045) which is gratefully acknowledged. One of us (C. Rajchenbach) wants also to thank DRET for the attribution of a grant fellowship.

\section{REFERENCES}

[1] Delpuech A., J. de Physique, C34-48 (1987) 353.

Odiot S., J. de Physique, C34-48 (1987) 225.

[2] Akhmanov S.A. and. Koroteev N.I, Methods of ivonlinear Optics in Light Scattering Spectroscopy

(Nauka, Moscow, 1981, in Russian).

[3] Shen Y.R., The Principles of Non-linear Optics, (Wiley, New York 1984).

[4] Shkurinov A. P., Jonusauskas G., Rullière C., J. Ram. Spectroscopy, 25 (1994) 359.

[5] Hébert P., Marguet S., Gustavsson T., Mialocq J.C., Opt. Comm., 90 (1992) 85

[6] Cataliotti R. S., Foggi P., Giorgini M. G., Mariani L., Morresi A. and Paliani G.,

J. Chem. Phys., 98 (1993) 4372.

[7] Létard J. F., Dumon P., Jonusauskas G., Dupuy F., Pée Ph., Rullière C., Lapouyade R.,

J. Phys. Chem. 98 (1994) 10391.

[8] Shkurinov A. P., Koroteev K., Jonusauskas G., Rullière C., Chem. Phys. Lett., 223 (1994) 573.

[9] Shipp K. G., J.Org.Chem., 29 (1964) 2620.

[10] Mc Gookin A., J. Soc. Chem. Ind., 68 (1949) 195.

[11] Lau A., Werncke W., Pfeiffer M., Spectrochimica Acta Rev., 13 (1990) 191.

[12] Goldberg L. S., Picosecond Phenomena III, Editors K. B. Eisenthal, R. M. Hochstrasser, W. Kaiser,

A. Laubereau, (Springer Berlin, 1982) p.94.

[13] Chikishev A. Yu., Kamalov V. F., Koroteev N. I., Kvach V. V., Shkurinov A. P., Toleutaev B. N., Chem. Phys. Lett., 144 (1988) 90.

[14] Matsunuma S., Akamatsu N., Kamisuki T., Adachi Y., Maeda S., Hirose C.,

J. Chem. Phys., 88 (1988) 2956.

[15] Werncke W., Lau A., Pfeiffer M., Weigmann H. J., Freyer W., Tschö J. T., Kim M. B.,

Chem. Phys., 118 (1987) 133.

[16] Kamalov V. F., Koroteev N. I., Toleutaev B. N. in Time Resolved Spectroscopy,

Editors R. J. Clarck, R. E. Hester, (John Wiley and Sons Ltd, New York) 1989 p. 255.

[17] Nagakura S., Mol. Phys. 3 (1960) 152. 
[18] Rajchenbach C., Jonusauskas G., Rullière C., Chem. Phys. Lett. (in press).

[19] Schoen P. E., Marrone M. J., Schnur J. M., Goldberg L. S., Chem. Phys. Lett., 90 (1982) 272.

[20] Sukowski U., Seilmeier A., Elsaesser T., Fischer S. F., J. Chem. Phys., 93 (1990) 4094.

[21] Elsaesser T., Kaiser W., Annu. Rev. Phys. Chem., 42 (1991) 83.

[22] in Handbook of Chemistry and Physic, CRC Press, Ed. D.R. Lide, (1992-1993) 73rd edition.

[23] Rajchenbach C., Jonusauskas G., Dumon P.,Rullière C., (in preparation for J. Energ. Mat.).

[24] Greene B. I., Hochstrasser R.M., Weisman R. B., J. Chem. Phys. 70 (1979) 1247.

[25] Chandra B. P., Ramrakhiani M., Ansari M. H., Tiwari S., Pramana J. Phys. 36 (1991) 407.

[26] Chandra B. P., Rahangdale Y., Ramrakhiani M., Phys. Stat. Solid. A 121 (1990) 281.

[27] Chandra B. P., Rahangdale Y., Cryst. Res. Technol. 25 (1990) 197.

[28] Tokhmetov A. T, Vettegren' V. I., Sov. Phys. Solid. State 31 (1989) 2125. 